\title{
Multiscale verification calculations for regional ensemble forecasts
}
D. Smith ${ }^{1}$
M. Naughton ${ }^{2}$
A. Sulaiman ${ }^{3}$

(Received 28 January 2011; revised 13 October 2011)

\begin{abstract}
Verification calculations are applied to selected data from an Australian region ensemble forecast, in both standard and multiscale formats. The scale partitioning, provided by a discrete wavelet transform, offers additional insights into the forecast error behaviour.
\end{abstract}

\section{Contents}

1 Introduction

2 Numerical methods

2.1 Governing equations and discretisation . . . . . . . . . .

2.2 Global and regional forecast ensembles . . . . . . . . .
C883

C884

C884

C884

http://journal.austms.org.au/ojs/index.php/ANZIAMJ/article/view/3936 gives this article, (c) Austral. Mathematical Soc. 2011. Published October 20, 2011. ISSN 1446-8735. (Print two pages per sheet of paper.) Copies of this article must not be made otherwise available on the internet; instead link directly to this URL for this article. 
3 Calculated results

C885

3.1 A regional forecast for zonal wind at $850 \mathrm{hPa} \ldots \ldots$. . . . C885

3.2 Ensemble verification . . . . . . . . . . . . . . . . C885

3.3 Multiscale verification . . . . . . . . . . . . . C890

4 Summary and conclusions

C894

References

C895

\section{Introduction}

Spatial discretisation of unsteady partial differential equations yields dynamical systems with unspecified initial conditions. For numerical weather prediction purposes, computationally intensive data assimilation calculations [14] provide initial condition approximations, which are inevitably laced with uncertainty from various sources [15]. Errors are thus present from the outset, and subsequently propagate during a forecast under a range of model deficiencies and numerical inaccuracies. Ensembles address this uncertainty using a family of perturbed forecasts, an instructive example being the "poor man's ensemble', which exploits the diversity of models, discretisations and initial conditions available from different forecasting centres around the world [8]. Diversity is strictly confined to the initial conditions in this ensemble study, which involves forecasts up to three days ahead, over the Australian region.

As with any forecast, ensembles are subject to verification calculations [5], which have recently been adapted to account for the inherent multiscale nature of atmospheric flows $[12,13]$. Synonymous with multiple scales is the wavelet decomposition, used by Briggs and Levine [5] to provide an objective data partitioning from which a corresponding set of verification scores was calculated, thus addressing a perceived inadequacy of univariate scores. In a variation on this multiscale theme, which treats atmospheric field data as an image, this study performs wavelet partitioning along meridional segments in 
an attempt at multiscale verification, offering some useful insights.

\section{Numerical methods}

\subsection{Governing equations and discretisation}

Working in spherical polar coordinates, the underlying conservation equations describe compressible non-hydrostatic atmospheric flow, subject to spatial discretisation by finite differencing on a regular latitude-longitude grid, and variable resolution terrain following vertical coordinates [7]. In conjunction with this is a two level predictor-corrector temporal integration scheme, implemented in a semi-implicit, semi-Lagrangian formulation for all equations except continuity, which is handled in Eulerian form. At the heart of each timestep is an iterative Helmholtz solver for the pressure increment, using a preconditioned generalised conjugate residual method [20]. Alternative atmospheric models in current use employ a range of different grids and discretisation schemes, including spectral elements on cubed spheres [11], spherical harmonic expansions [2], and spherical geodesic grids [16].

\subsection{Global and regional forecast ensembles}

A component of the Australian Community Climate and Earth Simulator System (ACCESS) numerical weather prediction suite [19], the global and regional ensemble prediction system (AGREPS) [17] is an Australian version of the North Atlantic-Europe local area ensemble of the UK Met Office [4]. The Australian regional domain operates on a $220 \times 320$ latitude-longitude mesh with 50 vertical levels and horizontal resolution of just under $40 \mathrm{~km}$. The global host ensemble, working on a $217 \times 288$ latitude-longitude mesh of coarser resolution $(90 \mathrm{~km})$, supplies initial and lateral boundary conditions to the regional ensemble via interpolation in space and time. Alternative 
approaches to regional domains, avoiding potential interpolation issues, use local mesh refinement of a global calculation, as applied in stretched grids [9], and spectral elements [11].

Initial condition perturbations are generated in the global ensemble with an Ensemble Transform Kalman Filter [1, 17], ETKF, and subsequently mapped to the regional domain. The ETKF transforms a set of forecast perturbations to a corresponding set of analysis perturbations, thus updating the ensemble in preparation for a new forecast, on 12 hour cycles in this system. Atmospheric observations, from numerous sources, play a key role in this method, unlike the popular singular vector approach, which identifies directions of most rapid error growth in the associated linearised model dynamics [15].

\section{Calculated results}

\subsection{A regional forecast for zonal wind at $850 \mathrm{hPa}$}

Visualising a 24 member ensemble forecast presents a challenge, addressed in Figure 1 by comparing the ensemble mean with its control (unperturbed) member, for a forecast of zonal wind at $850 \mathrm{hPa}$ (approximately $1500 \mathrm{~m}$ above sea level), or U850. This view is chosen to demonstrate the known smoothing effect of ensemble averaging, attributed to a nonlinear filtering process which acts to cancel errors from individual members [21]. Subsequent verification calculations pose further challenges in compressing large volumes of data to a manageable number of appropriate indicators.

\subsection{Ensemble verification}

Verification calculations [5] involve the comparison of forecast predictions for selected atmospheric quantities with like values from a verifying source, such as observations or atmospheric analyses. The latter case represents the best 

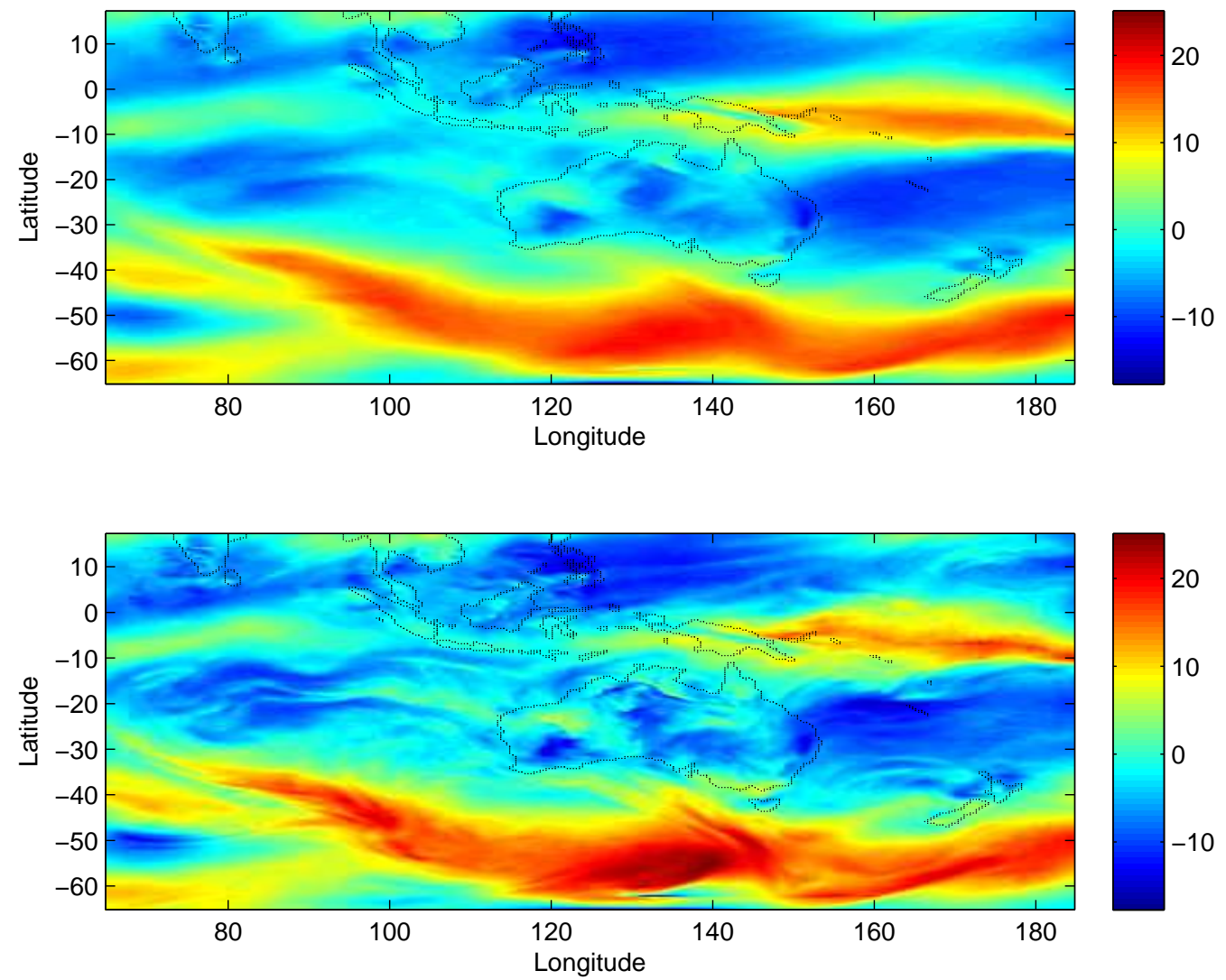

Figure 1: Ensemble mean for a three day U850 forecast, started at 1800 UTC on $04 / 02 / 2010$, in the upper panel, accompanied by the control member, demonstrating a smoothing effect. 

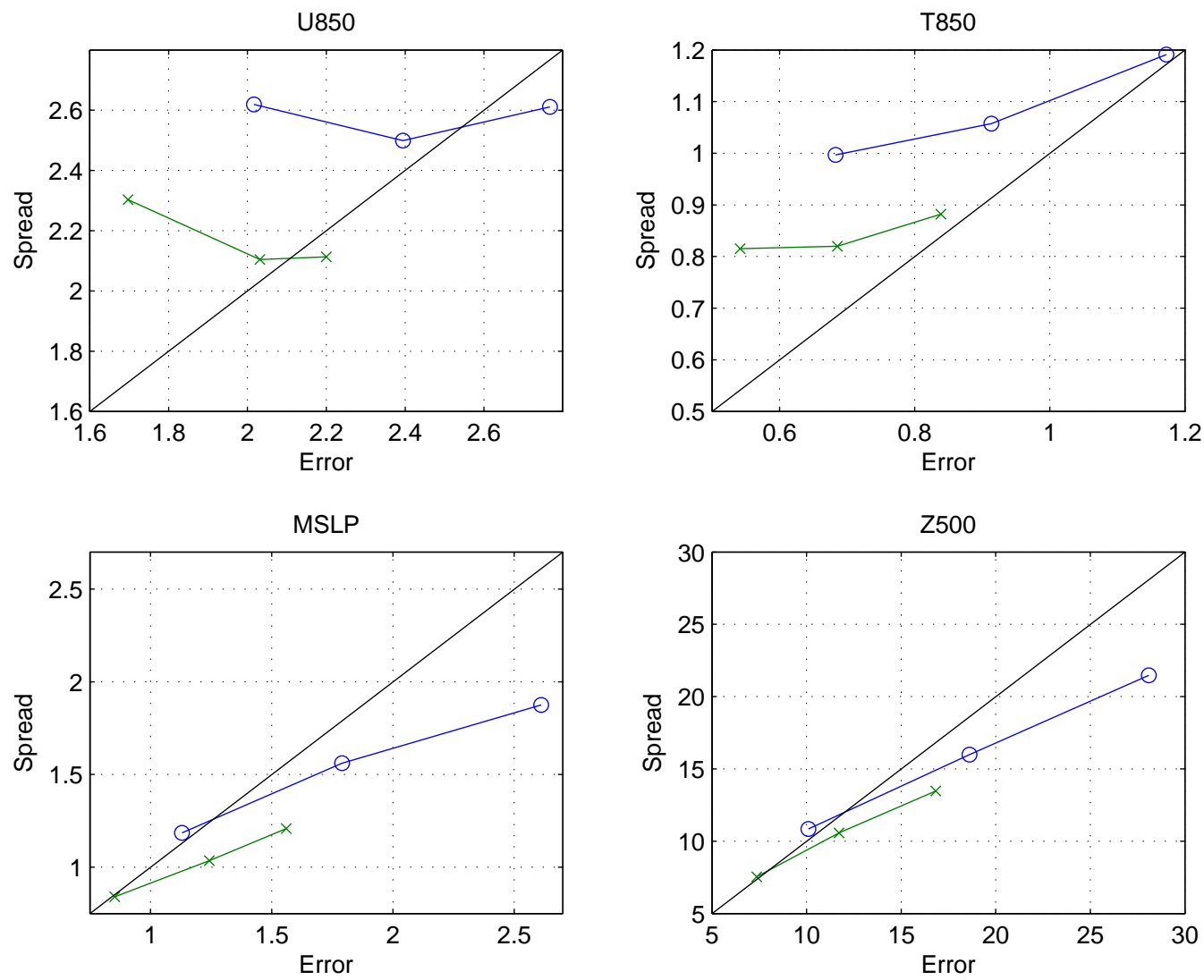

Figure 2: Spread-error plots for selected quantities forecast up to three days from 1800 UTC on 04/02/2010, with $\times$ denoting latitude weighting, producing qualitatively similar behaviour in each case.

available estimate of the atmospheric state at verification time, as supplied by the data assimilation process [14], and is used as the verification source here. Two key attributes of an ensemble are spread and skill, reflecting its dispersion (uncertainty) and accuracy, respectively. For spread, the ensemble root mean square deviation about its own mean provides a value at every gridpoint, while 
departure of the ensemble mean from the verification serves as a corresponding skill or error measure [15]. Taking root mean squared values of these quantities across the domain provides representative values for visualisation in spreaderror plots. Latitude weighting is often applied in these circumstances, in order to compensate for grid shrinkage at high latitudes [12], and subsequent results provide both weighted and unweighted versions for comparison.

Ensembles strive to achieve parity between spread and skill, guided by the unattainable perfect case of an ensemble mean agreeing with its verification at every gridpoint, which provides a useful reference indicator for spread [3, 12]. Accordingly, the spread-error plots of Figure 2 display both under and over spreading during the three day forecast, with overall error growth far exceeding the spread variation in each case. The U850 forecast becomes under spread on day three, while temperature at $850 \mathrm{hPa}$ (T850) approaches the diagonal from above. Mean sea level pressure (MSLP) and $500 \mathrm{hPa}$ geopotential height (z500) qualitatively resemble one another by diverging from the diagonal on the underspread side. The application of latitude weighting essentially shifts the curves downwards in each case, indicating an earlier underspreading.

Over and under spreading of ensembles remains a regular occurrence, receiving constant attention in the literature. Spread-skill analyses for a global 51 member singular vector ensemble, verified against analyses [12], indicated over spreading for the first four days of a 15 day forecast, followed by a transition to under spreading, for the quantity Z500. Verifying against observations, the ETKF results of Bowler et al. [3] reported good spread-skill matching for MSLP accompanied by under spreading of windspeed at $850 \mathrm{hPa}$, once again in a global ensemble. Both these studies pointed to the construction of initial condition perturbations as culpable for the observed mismatch, underlining the need for ongoing research in this discipline. 

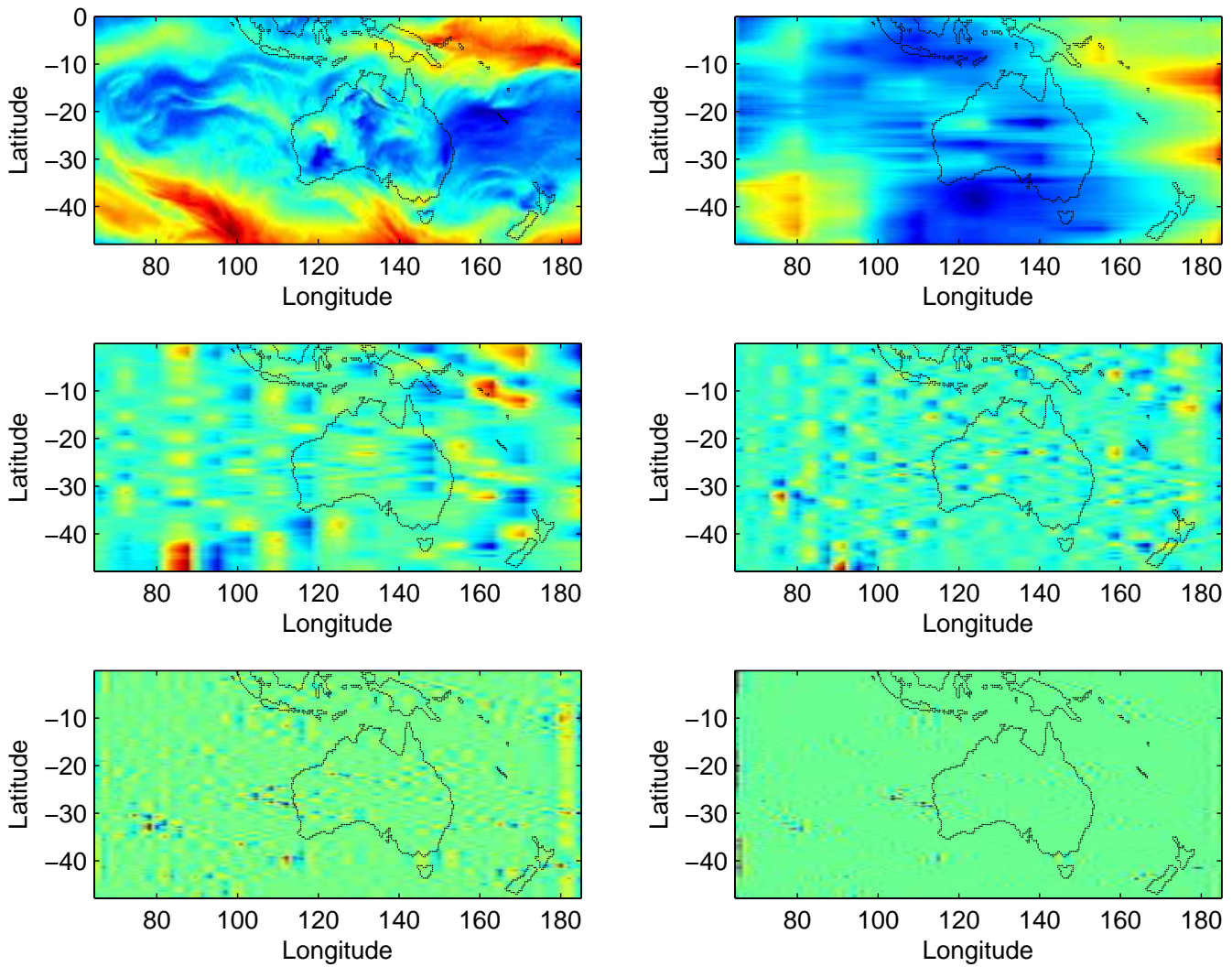

Figure 3: $\mathrm{D}_{2}$ components for the U850 control forecast from Figure 1. At the top left is the original reduced domain data, followed by the coarse approximation at the top right, then finer scale components on levels three to six, moving left to right and down the figure. In this case the coarse approximation and level three component combine to recover over $95 \%$ of the original data. 


\subsection{Multiscale verification}

Atmospheric flows are characterised by a multitude of scale specific flow phenomena on a wide range of scales [13], stimulating recent verification studies in a multiscale framework. Jung and Leutbecher [12] applied spectral filtering to isolate planetary, synoptic and sub-synoptic wavenumber bands in verification calculations on a global ensemble. They concluded that synoptic scales were the principal contributor for ensemble spread in short range forecasts up to four days, bearing adverse implications for the forecasting of atmospheric phenomena on these scales, such as extra-tropical cyclones. Wavelet decompositions offer an alternative spatial filtering approach based on a certain set of scales, dependent on the type of wavelet, which have previously been exploited in a number of atmospheric contexts. For blocking studies, one dimensional transforms were applied to pressure level data on latitude circles, unfolding into zonal scale and longitude location indices [10]. Two dimensional wavelet transforms have been implemented by projecting atmospheric field data onto a plane grid [18], or by treating the data as an image [5].

For the forecast data under consideration, scale partitioning is sought via the Daubechies $\mathrm{D}_{2}$ orthonormal discrete wavelet decomposition, equipped with boundary adjustment for non-periodic data [6]. In this preliminary study, the transform is applied along meridians (N-S direction) of a reduced $128 \times 320$ subdomain, centrally located within the original domain, yielding a set of 128 transform coefficients for each longitude value. Latitude rows are not transformed in order to avoid the scale disparity between latitude circles of different size, in contrast to the two dimensional image approach of Briggs and Levine [5].

For a given data column $y$, containing $2^{\mathfrak{n}}=128$ elements, in the reduced domain, the inverse wavelet transform

$$
y=\sum_{k=0}^{2^{J}-1} \lambda_{J, k} \varphi_{J, k}+\sum_{j=J}^{n-1} \sum_{k=0}^{2^{j}-1} \gamma_{j, k} \psi_{j, k},
$$



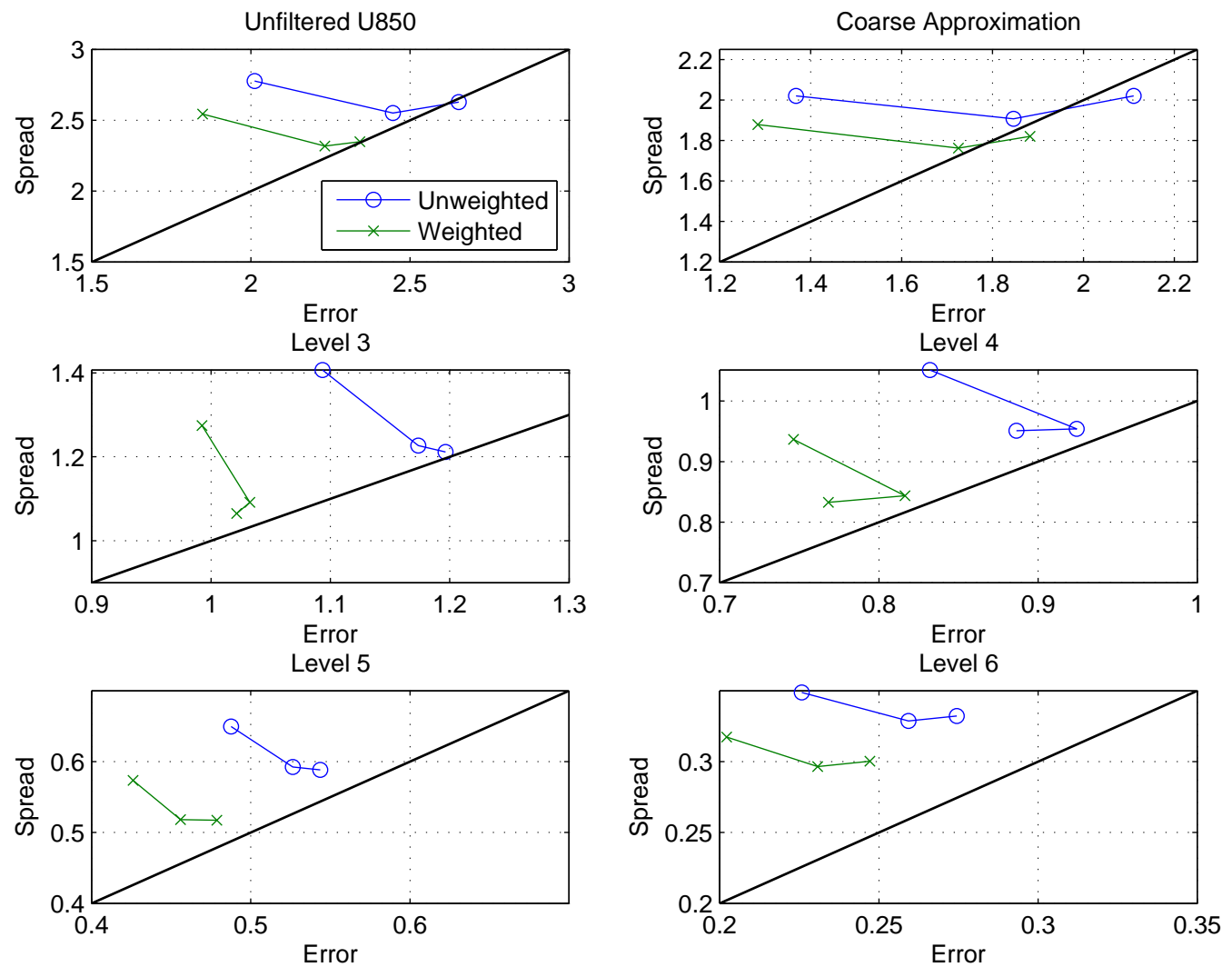

Figure 4: Multiscale spread-error plots for U850, based on $\mathrm{D}_{2}$ wavelet transform on meridional segments, with $\times$ indicating latitude weighting. 

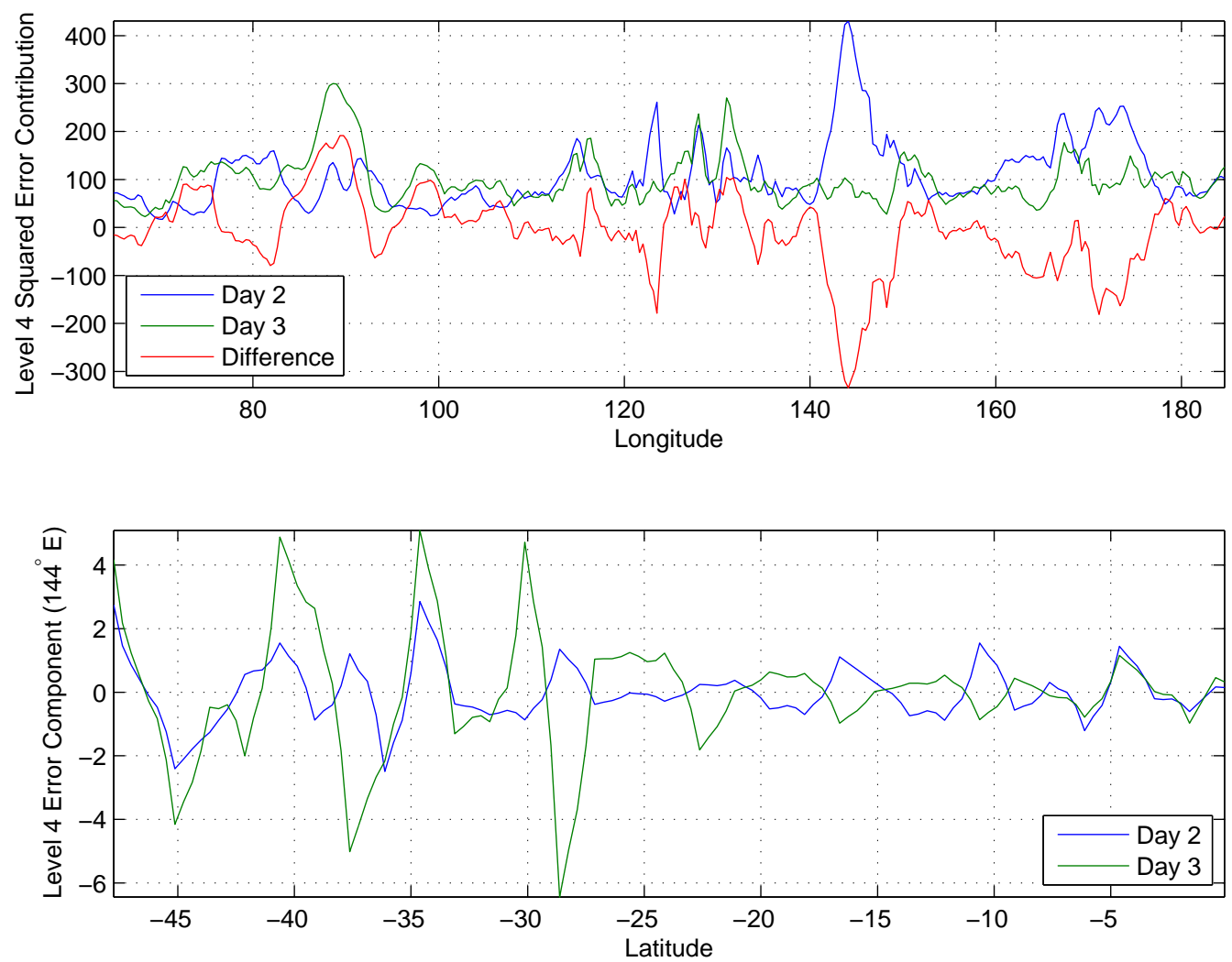

Figure 5: Level four $\mathrm{D}_{2}$ squared error contributions on days two and three for the U850 forecast, showing a large discrepancy near $145^{\circ}$, with associated error components near this longitude appearing in the lower plot. 
separates $y$ into a coarse approximation furnished by the scaling functions $\varphi_{\mathrm{J}, \mathrm{k}}$, and a set of components on progressively finer scales provided by the wavelets $\psi_{j, k}$ on the set of scales indexed by $j=J, \ldots, n-1$. The index $k$ denotes location, or latitude, for each scale, as distinct from wavenumber domain methods that involve a single index for spatial frequency [12]. For a data length of 128 , with bottom resolution level $J=3$, the expansion (1) offers four wavelet scales spanned by 8, 16, 32 and finally 64 wavelets on the top level. After transforming the entire ensemble forecast into the wavelet domain, effectively projecting it onto an orthonormal basis, application of (1) then unfolds the ensemble into five components, comprising a coarse approximation in the synoptic scales and four levels of finer detail. At the top level, spanned by 64 wavelets, this amounts to approximately $80 \mathrm{~km}$, increasing to 160,320 and finally 640 on level three, with eight wavelets. Applying (1) to the U850 control forecast data from Figure 1 produces the set of scale components displayed in Figure 3, in which the first two components recover over $95 \%$ of the original data.

Under this particular decomposition, the ensemble means for all forecasts represented in Figure 2 are strongly dominated by their coarse approximations, corresponding to synoptic scale features along meridians. As in previous wavelet and wavenumber domain approaches [5, 12], forecasts and their associated verifying analyses are separated into their respective scale components via (1), providing a new set of ensemble data on which the same spread and skill calculations are subsequently performed.

Figure 4 gives a new set of spread-error plots for U850, derived from the $\mathrm{D}_{2}$ transform operation, comprising the unfiltered result with its five filtered counterparts. The coarse approximation essentially follows the unfiltered result, crossing the diagonal to become underspread on day three, reflecting the dominance of this component in the ensemble mean for each forecast day. For the finer scale details, all four cases qualitatively mirror the coarse approximation behaviour on days one and two, approaching the diagonal with simultaneous spread decline and error increase, before some obvious departures emerge on day three. Most conspicuous of these is the error 
reversal (5-10\%) evident on level four, in which an error decrease accompanies a slight spread decrease. The remaining levels all feature an error increase, with associated spread increasing on level six, and declining on levels three and five.

The error reversal observed on level four in Figure 4 invites closer scrutiny, afforded by the corresponding squared error contributions for days two and three, given in Figure 5. Comparing the two curves reveals a prominent local peak centred near $145^{\circ}$ on day two, generating the largest decrease between the two days. Subsequent reconstruction of the associated wavelet error components near this longitude further localises the responsible activity to poleward of $25^{\circ}$ south, by virtue of large amplitude discrepancies. This type of localisation, in both space and scale, is expected to support the diagnosis of possible model deficiencies.

\section{Summary and conclusions}

Selected atmospheric fields from a three day regional ensemble forecast, with initial condition perturbations supplied by an ensemble transform Kalman filter, have been verified against analysis data. As a first attempt at multiscale verification, spread-skill calculations have been supplemented by multiscale versions derived from a discrete wavelet transform applied along meridional segments, unfolding the forecast into five components. A focus on the zonal wind at $850 \mathrm{hPa}$ has indicated signs of scale dependence in the verification process, providing an impetus for further exploration in this direction. Of particular interest in this regard is the emergence of a distinct error decline on one particular scale, which was subsequently traced to a locally large decrease in the ensemble mean error contribution at that scale, localised in space by the wavelet decomposition. Although preliminary in nature, such results can potentially assist in the diagnosis and correction of model deficiencies. Alternative wavelet decompositions possessing different resolution properties in the space-wavenumber domain deserve consideration, ultimately leading 
to spherical wavelets. Future studies will incorporate model perturbations, including a mechanism to counter kinetic energy loss through numerical advection error and diffusion.

\section{References}

[1] C. Bishop, B. Etherton and S. Majumdar, Adaptive Sampling with the Ensemble Transform Kalman Filter. Part I: Theoretical Aspects, Mon. Wea. Rev. 129 pp.420-436 (2001). doi:10.1175/1520-0493(2001)129;0420:ASWTET¿2.0.CO;2 C885

[2] W. Bourke, A Multi-Level Spectral Model. I. Formulation and Hemispheric Integrations, Mon. Wea. Rev. 102 pp.687-701 (1974). doi:10.1175/1520-0493(1974)102j0687:AMLSMI_2.0.CO;2 C884

[3] N. Bowler, A. Arribas, S. Beare, K. Mylne and G. Shutts, The local ETKF and SKEB: Upgrades to the MOGREPS short-range ensemble prediction system, Q.J.R. Meteorol. Soc. 135 pp.767-776 (2009). doi:10.1002/qj.394 C888

[4] N. Bowler, A. Arribas et al., The MOGREPS short-range ensemble prediction system, Q.J.R. Meteorol. Soc. 134 pp.703-722 (2008). doi:10.1002/qj.234 C884

[5] W. Briggs and R. Levine, Wavelets and Field Forecast Verification, Mon. Wea. Rev. 125 pp.1329-1341 (1997). doi:10.1175/1520-0493(1997)125;1329:WAFFV ¿2.0.CO;2 C883, C885, C890, C893

[6] A. Cohen, I. Daubechies and P. Vial, Wavelets on the Interval and Fast Wavelet Transforms, Applied and Computational Harmonic Analysis, 1 pp.54-81, (1993). doi:doi:10.1006/acha.1993.1005 C890 
[7] T. Davies, M. Cullen, A. Malcolm, M. Mawson, A. Staniforth, A. White and N. Wood, A new dynamical core for the Met Office's global and regional modelling of the atmosphere, Q.J.R. Meteorol. Soc. 131 pp.1759-1782 (2005). doi:10.1256/qj.04.101 C884

[8] E. Ebert, Ability of a Poor Mans Ensemble to Predict the Probability and Distribution of Precipitation, Mon. Wea. Rev. 129 pp.2461-2480 (2001). doi:10.1175/1520-0493(2001)129;2461:AOAPMS_2.0.CO;2 C883

[9] M. Fox-Rabinovitz, L. Takacs, R. Govindaraju and M. Suarez, A Variable-Resolution Stretched-Grid General Circulation Model: Regional Climate Simulation, Mon. Wea. Rev. 129 pp.453-469 (2001). doi:10.1175/1520-0493(2001)129;0453:AVRSGG_2.0.CO;2 C885

[10] A. Fournier, Atmospheric Energetics in the Wavelet Domain. Part II: Time-Averaged Observed Atmospheric Blocking, J. Atm. Sci. 60 pp.319-338 (2003).

doi:10.1175/1520-0469(2003)060ز0319:AEITWD¿2.0.CO;2 C890

[11] A. Fournier, M. Taylor and J. Tribbia, The Spectral Element Atmosphere Model (SEAM): High-Resolution Parallel Computation and Localized Resolution of Regional Dynamics, Mon. Wea. Rev. 132 pp.726-748 (2004).

doi:10.1175/1520-0493(2004)132j0726:TSEAMS¿2.0.CO;2 C884, C885

[12] T. Jung and M. Leutbecher, Scale-dependent verification of ensemble forecasts, Q.J.R. Meteorol. Soc. 134 pp.973-984 (2008). doi:10.1002/qj.255 C883, C888, C890, C893

[13] R. Klein, Scale-Dependent Models for Atmospheric Flows, Annual Review of Fluid Mechanics 42 pp.249-274 (2010). doi:10.1146/annurev-fluid-121108-145537 C883, C890

[14] A. Lawless, S. Gratton and N. Nichols, An investigation of incremental 4D-Var using non-tangent linear models, Q.J.R. Meteorol. Soc. 131 pp.459-476 (2005). doi:10.1256/qj.04.20 C883, C887 
[15] M. Leutbecher and T. Palmer, Ensemble Forecasting, J. Comp. Phys. 227 pp.3515-3539 (2008). doi:10.1016/j.jcp.2007.02.014 C883, C885, C888

[16] D. Majewski et al., The Operational Global Icosahedral-Hexagonal Gridpoint Model GME: Description and High-Resolution Tests, Mon. Wea. Rev. 130 pp.319-338 (2002). doi:10.1175/1520-0493(2002)130ز0319:TOGIHG_2.0.CO;2 C884

[17] T. O'Kane, M. Naughton and Y. Xiao, The Australian community climate and earth system simulator global and regional ensemble prediction scheme. ANZIAM J. 50 (CTAC2008) pC385-C398 (2008). http://journal . austms.org. au/ojs/index.php/ANZIAMJ/article/ view/1421 C884, C885

[18] M. Plu, P. Arbogast and A. Joly, A Wavelet Representation of Synoptic-Scale Coherent Structures, J. Atm. Sci. 65 pp.3116-3138 (2008). doi:10.1175/2008JAS2618.1 C890

[19] K. Puri et al., Preliminary results from Numerical Weather Prediction implementation of ACCESS, CAWCR Research Letters, Issue 5 pp.15-22 (2010). cawcr.gov.au/publications/researchletters/ CAWCR_Research_Letters_5.pdf C884

[20] P. Smolarkiewicz and L. Margolin, Variational Solver for Elliptic Problems in Atmospheric Flows, Appl. Math. Comp. Sci. 4 pp.527-551 (1994). http://nldr.library.ucar.edu/repository/collections/ PUBS-000-000-001-031 C884

[21] D. Stensrud and H. Brooks, Using Ensembles for Short-Range Forecasting, Mon. Wea. Rev. 127 pp.433-446 (1999). doi:10.1175/1520-0493(1999)127;0433:UEFSRF ¿2.0.CO;2 C885 


\section{Author addresses}

1. D. Smith, Centre for Australian Weather and Climate Research, Bureau of Meteorology, Melbourne Australia. mailto:dhsmith@bom.gov. au

2. M. Naughton, Centre for Australian Weather and Climate Research, Bureau of Meteorology, Melbourne Australia.

3. A. Sulaiman, Centre for Australian Weather and Climate Research, Bureau of Meteorology, Melbourne Australia. 